\title{
Neurovascular Decompression with Selective Partial Rhizotomy of the Trigeminal Nerve for Tic Douloureux
}

\author{
Steven E. Swanson, M.D., ${ }^{*}$ and Saeed M. Farhat, M.D.
}

Fourteen patients with classic tic douloureux were treated with neurovascular decompression of the trigeminal nerve at its zone of entry into the pons. In addition, selective rhizotomy of the portio major was performed in each patient. This combined procedure gave excellent relief of pain with no recurrences in a 23 -month postoperative period. Technical observations and postoperative complications in 3 patients are discussed. Routine selective partial rhizotomy of the sensory root of the trigeminal nerve may be a useful adjunct to neurovascular decompression for tic douloureux.

Swanson SE, Farhat SM: Neurovascular decompression with selective partial rhizotomy of the trigeminal. nerve for tic douloureux. Surg Neurol $18: 3-6,1982$

A number of authors have noted that compression of the trigeminal nerve by vascular structures in the posterior fossa plays a significant role in the etiology of tic douloureux [3, $4,7]$. Neurovascular decompression of the trigeminal nerve at its entry zone, as popularized by Rand and Jannetta, has become a well-accepted procedure for tic douloureux and can be performed with minimal morbidity and mortality [7, $8,10]$. Since the pathophysiology of tic douloureux is not completely understood, and since a compressive vascular structure cannot be found in all cases, selective partial rhizotomy in conjunction with neurovascular decompression was used in an effort to partially denervate the most frequently encountered cutaneous trigger zone and, thus, prevent early postoperative recurrence of tic douloureux pain. This report summarizes the recent experience of the senior author (S. M. F.) using neurovascular decompression with selective partial rhizotomy of the sensory root of the trigeminal nerve to treat patients with tic douloureux.

*Section of Neurosurgery, University of Michigan Hospital, 1405 East Ann St., Ann Arbor, MI 481C9.

From the Section of Neurosurgery, Department of Surgery, University of Michigan Hospital, and St. Joseph Mercy Hospital, Ann Arbor, MI.

Address reprint requests to $\mathrm{Lr}$. Saeed M. Farhat, Professional Office Building, Suite 3B-35, 5305 East Huron River Dr., Ypsilanti, MI 48197.

Key words: tic douloureux; trigeminal nerve; thizotomy; neurovascular decompression.

\section{Clinical Materials and Methods}

Fourteen patients ( 5 men and 9 women) were treated for classic tic douloureux with neurovascular decompression and partial selective rhizotomy between March, 1979, and May, 1981. All of the patients had been treated with carbamazepine or phenytoin sodium, or both, and were either refractory to the effects of these medications or were unable to tolerate their side effects. Two of the patients had previously undergone radiofrequency neurolysis and infraorbital nerve avulsion for relief of their pain. The patients ranged in age from 22 to 74 years (average, 51 years). The duration of time that elapsed between onset of symptoms and a definitive neurosurgical procedure ranged from 3 months to 27 years and averaged 5.8 years. Pain occurred on the right in 10 patients and on the left in 4 . The distribution of pain among the 14 patients was as follows: second division (4 patients), third division ( 2 patients), and multiple divisions ( 8 patients); no patients had pain in the first division.

Preoperative evaluation included a general medical examination as well as a complete neurological examination. Other than their common complaint of tic douloureux, our patients were all in relatively good general health. In addition to a routine laboratory investigation, all patients underwent roentgenographic examination of the skull, with base views, and contrast-enhanced computerized tomography.

Approximately 12 hours before operation, all patients were given steroids, which were continued throughout the operative procedure and then decreased rapidly during the early postoperative period. A right atrial catheter was placed via either the subclavian or jugular vein and its position verified by preoperative roentgenographic examination of the chest.

\section{Operative Procedure}

General endotracheal anesthesia was administered, and patients were placed in a modified sitting position with the head and neck flexed and slightly rotated to the involved side using a pin-fixation head holder. Once proper positioning was attained, the atrial catheter was checked for ease of aspiration and a Doppler flowmeter was positioned over the precordium to monitor for air emboli.

A $3 \times 3 \mathrm{~cm}$ retromastoid suboccipital craniectomy was 


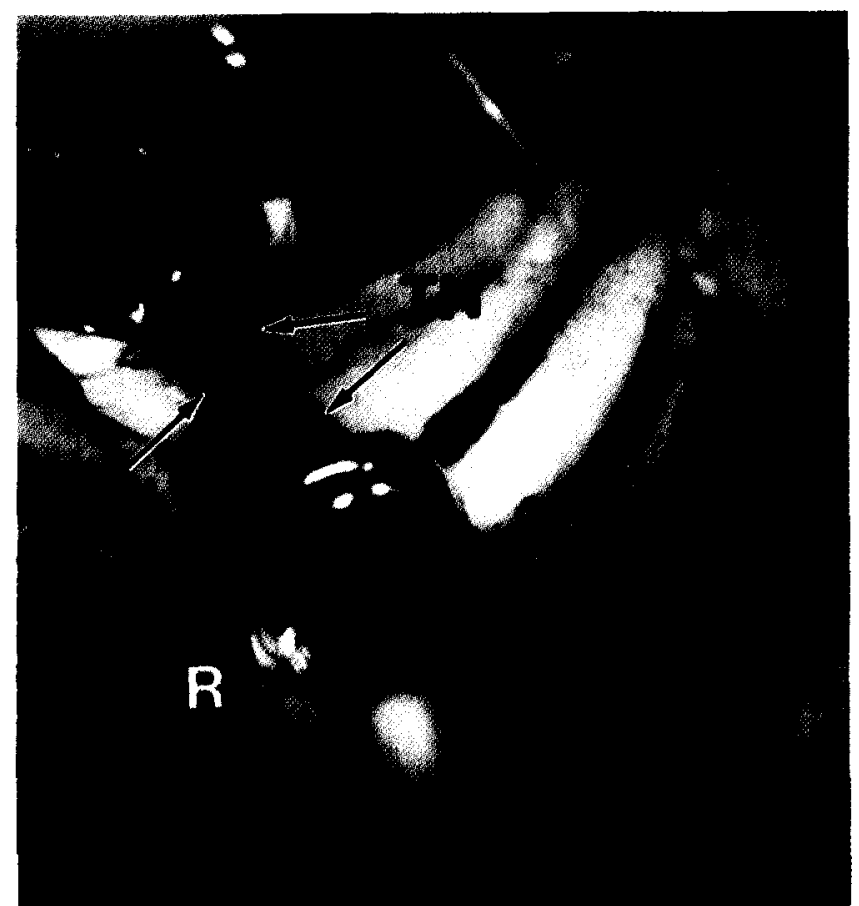

Fig. 1. Intraoperative photograph of the proximal right trigeminal nerve (TN). A branch of the superior cerebellar artery (arrows) is compressing the trigeminal nerve at its zone of entry into the brain stem. The superolateral aspect of the cerebellar hemisphere is beneath the retractor (R).

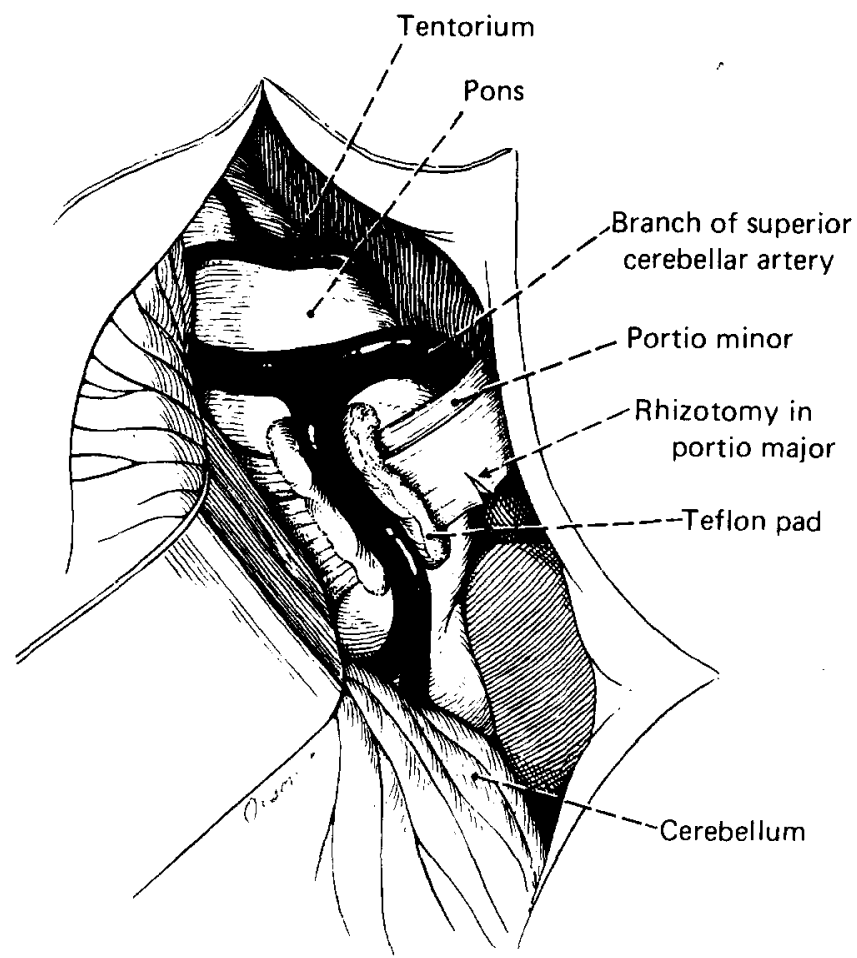

Fig. 2. Approximate location of the selective partial rhizotomy of the portio major. The posterolateral nerve fibers of the portio major are divided to the depth of $1 \mathrm{~mm}$ just distal to the entry zone of the right trigeminal nerve.

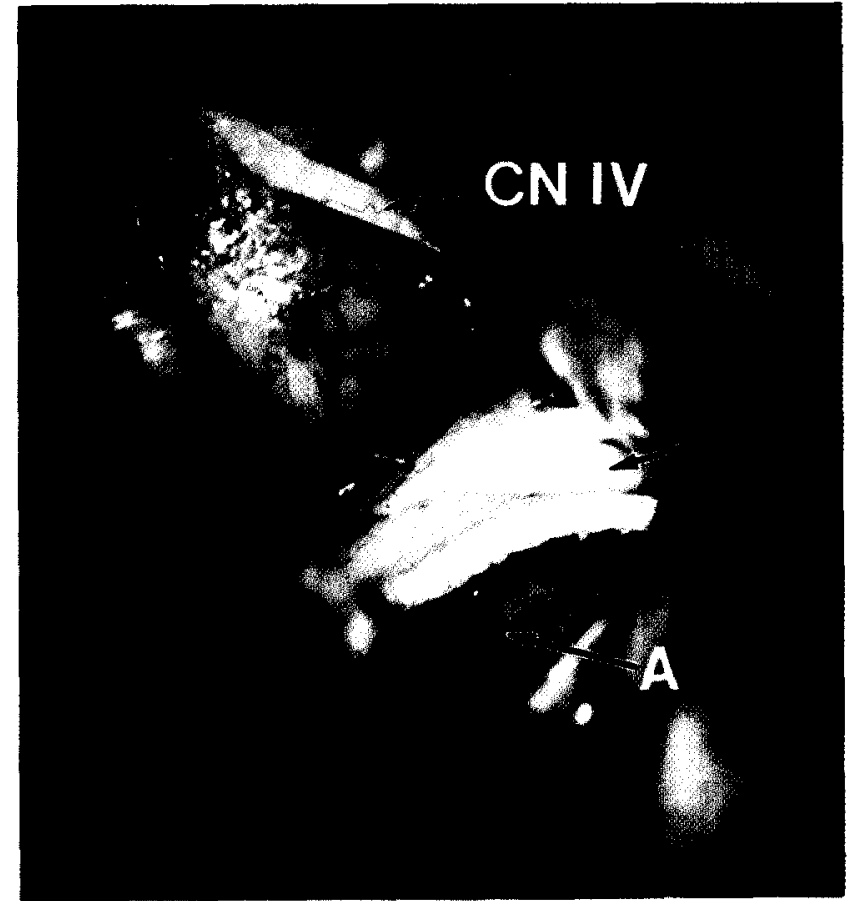

Fig. 3. Intraoperative photograph of the right trigeminal nerve after neurovascular decompression. The nerve (small arrow) is sandwiched between two Teflon sponges that elevate a branch of the superior cerebellar artery above and a fusiform aneurysm of the anterior inferior cerebellar artery (A) below. The trochlear nerve (CN IV) can be seen in the superior portion of the operative field.

A $3 \times 3 \mathrm{~cm}$ retromastoid suboccipital craniectomy was performed through a $6 \mathrm{~cm}$ paramedian incision that extended approximately $2 \mathrm{~cm}$ above the superior nuchal line. Additional calvarium was rongeured away as necessary to obtain adequate exposure of the transverse and sigmoid sinuses. After the exposed bone edges and mastoid air cells were waxed, the dura mater was opened using a $Y$ incision, based inferiorly. The superolateral limb of the dural incision extended to the junction of the transverse and sigmoid sinuses. These dural leaflets were then reflected superiorly and laterally, respectively, to facilitate adequate exposure of the affected trigeminal nerve.

Gentle retraction on the superolateral aspect of the cerebellar hemisphere using a self-retaining retractor allowed placement of the surgical microscope (Zeiss Opmi, $300 \mathrm{~mm}$ objective). Once the superior petrosal vein was identified and divided, the retractor was repositioned to expose the trigeminal nerve. The arachnoid surrounding the trigeminal nerve was gently opened and dissected free from the nerve, allowing cerebrospinal fluid to drain from the paramesencephalic cistern. The arachnoid dissection was limited only to that area directly overlying the trigeminal nerve. The compressing blood vessel could often be seen through the arachnoid membrane and was dissected free from the nerve at the nerve's entry zone into the pons (Fig. 1). These arteries (branches of either the superior cerebellar 
artery or the anterior inferior cerebellar artery) or veins were prevented from migrating back into their former position by an interposed Teflon sponge, which had been fashioned to fit between the vessel and the nerve.

The proximal portio major and the intermediate fascicles were identified at the root entry zone of the trigeminal nerve. Selective partial rhizotomy of the most posterior fibers at the middle portion of the portio major was performed using microscissors, taking care not to injure the motor-proprioceptive intermediate fascicles. The fibers were divided to a depth of $1 \mathrm{~mm}$ on the posterolateral aspect of the portio major, approximately $2 \mathrm{~mm}$ from its entrance into the brain stem (Fig. 2). The dura mater was reapproximated using a running 4.0 silk suture; a piece of Gelfoam was fashioned to fit into the craniectomy defect. The incision was closed in layers and covered with a sterile dressing.

\section{Results}

Findings at the time of operation revealed compression of the trigeminal nerve by branches of either the superior cerebellar or anterior inferior cerebellar arteries in 12 of the 14 patients. In 2 patients, the only offending vessel identified was a venous branch crossing the nerve at its entry zone. A cirsoid aneurysm of the anterior inferior cerebellar artery was found to be compressing the inferior aspect of the trigeminal nerve at its entry zone in 1 of the patients (Fig. 3).

Results have been excellent in 12 patients (no facial pain, no sensory loss, and no medication required) and good in 2 patients (occasional minor facial pain with little or no medication required) after postoperative observations of from 10 to 23 months. Thirteen of the patients have been followed for at least one year after their operative procedure. None of the patients suffered significant postoperative sensory loss, hearing loss, cerebellar signs, or anesthesia dolorosa. No postoperative deaths occurred.

Complications occurred in 3 patients and included transient diplopia, tension pneumocephialus with intracerebral hematoma, and chronic subdural hematoma. Two of the 3 patients had trochlear nerve dysfunction with resulting superior oblique muscle palsy. Transient diplopia developed in 2 patients and resolved spontaneously and completely within two weeks after operation. Headache and hemiparesis developed in 1 patient approximately six months after his neurovascular decompression, and evacuation of a left frontal chronic subdural hematoma was tequired. Finally, 1 patient required an emergency reoperation in the early postoperative period to relieve tension pneumocephalus and to evacuate an intracerebral hematoma. Both patients who underwent reoperation made excellent neurological recoveries.

\section{Discussion}

Neurovascular decompression for tic douloureux, as described by Gardner [5,11], is a safe, nondestructive form of therapy for this disease. Several large series document that the procedure can be performed with excellent clinical improvement in the patients' symptoms and with minimal morbidity and mortality $[1,2,7,8,13]$. Our series corroborates the results of others in that all of our patients obtained substantial symptomatic relief of their pain, with minimal morbidity and no mortality. In large series, tumors and multiple sclerosis have been implicated as the cause of tic douloureux in about $12 \%$ of the patients [7]. In our more limited series, vascular compression of the trigeminal nerve at its entry into the pons was found in each patient.

While the operative exposure and neurovascular techniques have been reviewed elsewhere [7], several technical considerations warrant discussion. All of our patients were operated on in a modified sitting position which, when combined with drainage of cerebrospinal fluid from the paramesencephalic cistern, allowed excellent exposure of the trigeminal nerve at its entry zone. Drainage of a small amount of cerebrospinal fluid made exposure possible, with minimal cerebellar retraction, and obviated the routine intraoperative use of osmotic agents. This approach seems to minimize the amount of air that enters the subarachnoid space and makes persistent headache a much less commion cause of postoperative morbidity.

The absence of significant postoperative cerebellar symptoms or cranial nerve palsies in our patients is related to two important technical points in the operation. First, unless the dural incision is carried directly to the junction of the transverse and sigmoid sinuses, the dura mater cannot be reflected adequately to give exposure of the trigeminal nerve. The tendency is then to retract the cerebellar hemisphere medially and inferiorly to obtain exposure, and therefore to run the risk of causing postoperative cerebellar dysfunction. Secondly, when the arachnoid dissection is limited to the area directly overlying the trigeminal nerve, the intact arachnoid protects the fourth, seventh, and eighth cranial nerves from inadvertent intraoperative trauma.

The trigeminal nerve leaves the lateral pons and courses in an anterolateral direction to Meckel's cave. While the orientation of the dorsal root entry zone at the pons may be obliquely vertical or horizontal, the relationship between the portio major and the motor-proprioceptive fibers remains constant, with the portio major inferior and lateral to the motor-proprioceptive fibers. The most posterior fibers in the midportion of the portio major were sectioned in the rhizotomy (see Fig. 2).

The exact location of pain and temperature fibers in the retrogasserian trigeminal nerve remains somewhat controversial; however, Rand's microanatomical studies [9] support Dandy's observation that these fibers are localized in the portio major and are physically separate from those fibers mediating tactile sensation. Nerve stimulation studies under local anesthesia demonstrate second and third division evoked pain sensation when the dorsolateral edge of 
the trigeminal nerve (portio major) is stimulated near the brain stem [6]. Since the rhizotomy performed in our patients involved only a few fascicles of the portio major, a critical reduction in the number of nerve fibers, resulting in symptomatic sensory loss, did not occur. Also, only the most posterior fibers of the portio major are sectioned so that theoretically the rhizotomy would be ineffective in a patient with strictly first division pain because those fibers run in the anterior half of the portio major.

Selective rhizotomy of the portio major has been reported to be the procedure of choice when tic douloureux is caused by a plaque of multiple sclerosis at the entry zone or if the compressing blood vessel cannot be safely mobilized away from the nerve [7,12]. Sectioning of the portio major at the brain stem preserves tactile sensation as well as the corneal reflex [7]. Burchiel and associates [2] recently reported their experience with partial rhizotomy of the trigeminal nerve in patients who had a negative exploration at the time of suboccipital craniectomy or who suffered a recurrence of their trigeminal neuralgia after microvascular decompression. They noted no complications associated with partial rhizotomy and found that $80 \%$ of their patients who underwent partial rhizotomy, either as a primary or secondary procedure, had complete relief of their pain. All of the patients in our series underwent partial rhizotomy of the portio major as an adjunct to neurovascular decompression, and all of the patients awoke from the operation free of pain. No clinically significant postoperative sensory loss was associated with the procedure in any of our patients. However, most of the patients noted a mild subjective numbness near the nasolabial fold and corner of the mouth ipsilateral to the partial rhizotomy. While no discrete area of sensory loss could be demonstrated by objective testing, this sensation is most likely secondary to the partial rhizotomy of the portio major. The cutaneous trigger zone of many patients with tic douloureux is located near the upper lip and nasolabial fold, and perhaps the minimal denervation achieved with selective partial rhizotomy of the portio major interrupts the stimulus that can evoke a paroxysm of tic pain.

Both patients who experienced postoperative complications that required reoperation were elderly, and the lesion, delayed chronic subdural hematoma in 1 patient and tension pneumocephalus with intracerebral hematoma in the other, may have been due to the operation being done with the patient in the sitting position. None of our patients have yet required reoperation for recurrent ipsilateral tic douloureux. Jannetta [8] had only 2 of his 450 patients suffer a recurrence of their tic douloureux when they had been free of pain for a year after their neurovascular decompression, but he reports an early recurrence rate of $11 \%$. None of our patients have suffered a recurrence of tic douloureux after neurovascular decompression with selective partial rhizotomy, with an average follow-up of 20 months. Further follow-up of a larger series of patients will be required to determine the efficacy of this procedure in permanently relieving the pain of tic douloureux.

In conclusion, the technique of neurovascular decompression of the trigeminal nerve has been shown to be a definitive and safe procedure when carried out by trained microneurosurgeons. The addition of selective partial rhizotomy of the portio major to the procedure potentiates immediate postoperative relief of pain and may reduce the chance of postoperative recurrence of pain without significantly increasing the morbidity or mortality associated with neurovascular decompression. Selective partial rhizotomy of the portio major becomes a particularly useful adjunct when compressing vessels are not found or are difficult to mobilize safely away from the nerve, or when multiple sclerosis is the apparent cause of the tic douloureux.

We are grateful to Miss Pat Frye, who typed this manuscript. Support for the preparation of this report was made possible through funds from the Section of Neurological Surgery, University of Michigan Hospital.

\section{References}

1. Apfelbaum RI: A comparison of percutaneous radiofrequency trigeminal neurolysis and microvascular decompression of the trigeminal nerve for the treatment of tic douloureux. Neurosurgery 1:16-21, 1977

2. Burchiel KJ, Steege TD, Howe JF, Loeser JD: Comparison of percutaneous radiofrequency gangliolysis and microvascular decompression for the surgical management of tic douloureux. Neurosurgery 9:111-119, 1981

3. Dandy WE: Concerning the cause of trigeminal neuralgia. Am J Surg $24: 447-455,1934$

4. Gardner WJ: Concerning the mechanism of trigeminal neuralgia and hemifacial spasm. J Neurosurg 19:947-958, 1962

5. Gardner WJ, Miklos MV: Response of trigeminal neuralgia to "decompression" of sensory root: discussion of cause of trigeminal neuralgia. JAMA 170:1773-1776, 1959

6. Hanraets PR: Surgical treatment of trigeminal neuralgia following the method of Dandy. Psychiatr Neurol Neurochir 73:441-446, 1970

7. Jannetta PJ: Microsurgical approach to the trigeminal nerve for tic douloureux. Prog Neurol Surg 7:180 -200, 1976

8. Jannetta PJ: Treatment of trigeminal neuralgia. Neurosurgery 4:93-94, 1979

9. Rand RW: Functional and anatomic localization in the trigeminal root: in support of Dandy, in Morley TP (ed): Current Controversies in Neurosurgery. Philadelphia: Saunders, 1976, pp 533-538

10. Rand RW: Microsurgical operations in the treatment of trigeminal neuralgia. J Microsurg 1:101-107, 1979

11. Rand RW: Gardner neurovascular decompression of the trigeminal and facial nerves for tic douloureux and hemifacial spasm. Surg Neurol 16:329-332, 1981

12. Waga $S$, Marikawa $A$, Kojima $T$ : Trigeminal neuralgia: compression of the trigeminal nerve by an elongated and dilated basilar artery. Surg Neurol 11:13-16, 1979

13. Wilson CB, Torke C, Prioleau G: Microsurgical vascular decompression for trigeminal neuralgia and hemifacial spasm. West J Med $132: 481-484,1980$ 